\title{
Analysis of the velocity distribution in different types of ventilation system ducts
}

\author{
Kazimierz Peszyński ${ }^{1,{ }^{*}}$, Lukasz Olszewski ${ }^{1}$, Emil Smyk ${ }^{1}$, and Daniel Perczyński ${ }^{1}$ \\ ${ }^{1}$ UTP University of Science and Technology in Bydgoszcz, Faculty of Mechanical Engineering, Control Group, Poland
}

\begin{abstract}
The paper presents the results obtained during the preliminary studies of circular and rectangular ducts before testing the properties elements (elbows, tees, etc.)of rectangular with rounded corners ducts. The fundamental problem of the studies was to determine the flow rate in the ventilation duct. Due to the size of the channel it was decided to determine the flow rate based on the integration of flow velocity over the considered cross-section. This method requires knowledge of the velocity distribution in the cross section. Approximation of the measured actual profile by the classic and modified Prandtl power-law velocity profile was analysed.
\end{abstract}

\section{Preliminary remarks}

The testing of ventilation systems components and their design can be greatly simplified by finding analytical solutions which determine the velocity distribution of air flow in a duct. Control Group of Mechanical Engineering Faculty UTP in Bydgoszcz in cooperation with Nucair Technologies Sp. z o.o., Solec Kujawski, Poland conducts research of new type ventilation ducts of rectangular with rounded corners cross-section. This duct type has significant operational advantages, but the aerodynamic phenomena occurring in these ducts have not been studied yet.

\section{Circular cross-section of duct}

In the state of art literature, the ventilation ducts with a circular cross-section are well examined in the terms of analysis. The ducts with rectangular cross-section are also well described. This paper presents the results of own research related to these two types of channels.

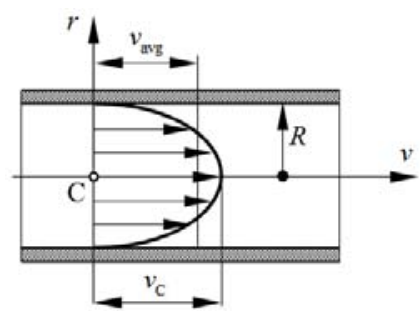

Fig. 1. Average velocity $v_{\text {avg }}$ for circular cross-section

During the study of pressure drop (hydraulic resistance), one of the main problems has been to determine the flow rate of air. These tests were aimed to determine the flow rate based on velocity distribution in a duct. In fluid flow, it is convenient to work with an average velocity $v_{\text {avg }}$ which remains constant in incompressible flow when the cross-section of the duct is constant (Fig. 1). A semi-empirical dependence was used due to the lack of accurate analytical solutions. It is known as Prandtl power-law velocity profile, which is expressed as $[1,2]$ for a duct of circular cross-section

$$
v=v_{\mathrm{C}}\left(1-\frac{r}{R}\right)^{\frac{1}{n}}
$$

where: $v, v_{\mathrm{C}}=v_{\max }, r, R$ are the quantities set out in Fig. 1, and $1 / n=1 / 6 \div 1 / 10$ is the exponent of the power for a range of Reynolds numbers $R e=4 \cdot 10^{3} \div 3,2 \cdot 10^{6}$ [1]. These values $1 / n$ refer to the smooth walls, and for the rough walls are $1 / n=1 / 4 \div 1 / 5$. Equation (1) expires near the wall.

\subsection{Analysis of the Prandtl power-law velocity profile}

The first doubts have risen during the numerical analysis of the problem in the ANSYS-FLUENT environment. The curve illustrating the velocity distribution along a diameter in Fig. 2 suggests that the tangent to velocity profile in the axis point of the circular cross-section duct is perpendicular to the axis. This can never be achieved when using the Prandtl approximation model, because after its differentiation with respect to $r$ we obtain

$$
\frac{\mathrm{d} v}{\mathrm{~d} r}=\left(v_{\mathrm{C}}\left(1-\frac{r}{R}\right)^{\frac{1}{n}}\right)^{\prime}=-\frac{1}{n R} v_{\mathrm{C}}\left(1-\frac{r}{R}\right)^{\frac{1}{n}-1}
$$

hence substituting into (2) $r=0$, we get

$$
\frac{\mathrm{d} v}{\mathrm{~d} r}=-\frac{v_{\mathrm{C}}}{n R} \neq 0
$$




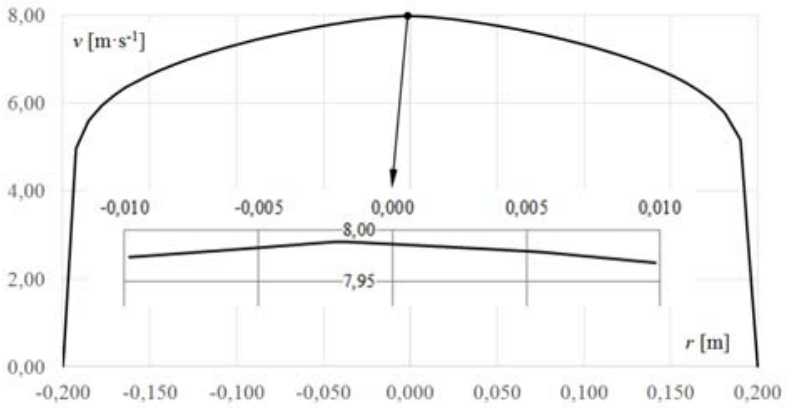

Fig. 2. Numerical solution in ANSYS-FLUENT

Because previously were studied rectangular ducts and rectangular ducts with rounded corners, where well they fits the modified Prandtl power-law velocity profile in the form

$$
v=v_{\mathrm{C}}\left(1-\left(\frac{r}{R}\right)^{2}\right)^{\frac{1}{n}}
$$

for the comparative analysis of the ducts shapes was adopted precisely this equation. Earlier, a comparison of velocity distributions of the both equations was done. The results of this analysis are shown in Fig. 3

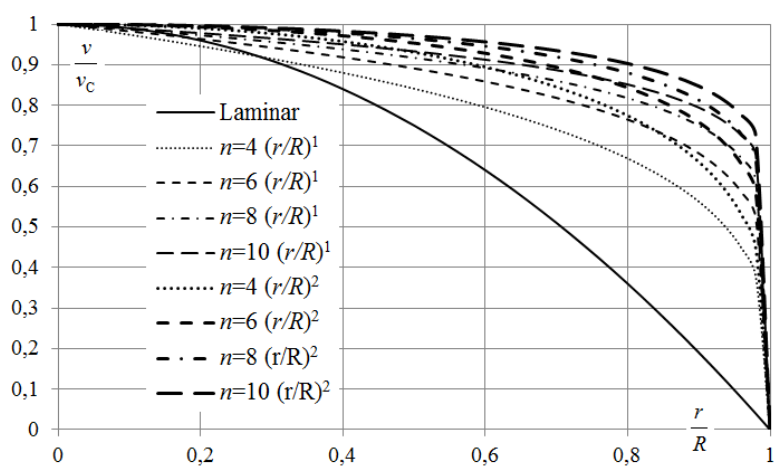

Fig. 3. Velocity distribution for fully developed flow

The figure Fig. 3 shows that the difference of distribution decreases with the increase of $n$. We can use smaller parameter $n$ by using distribution equation which includes $(r / R)^{2}$. Thanks to this operation, the function of distribution $v(r)$ will be the same for all types of ducts.

\subsection{Analysis of the tests results}

The study involved a linear section of the duct composed of 12 same segments with a radius $R=0,2 \mathrm{~m}$ and length $1 \mathrm{~m}$. The measurements were performed along the horizontal diameter. Fig. 4 presents the results of measurements and the approximation of this course by the two methods mentioned above. The approximation in this case turned out to be better by classic Prandtl equation. This conclusion has been drawn from the analysis of the sum of squares of deviations $\Delta=\left(v_{\mathrm{a}}-v_{\mathrm{m}}\right)^{2}$ for all measuring point.

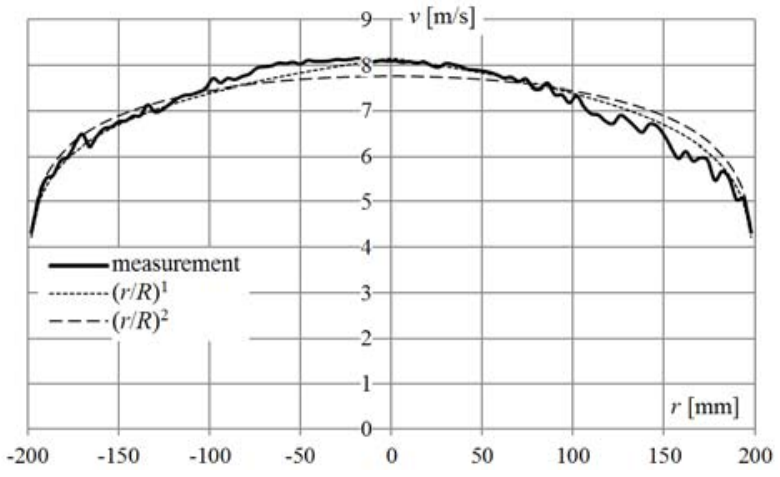

Fig. 4. Velocity distribution in circular cross-section

The maximum velocity on the duct axis determined on the basis of 20 measurements made with an interval of $1 \mathrm{~s}$ was $v_{\mathrm{Cm}}=8,11 \mathrm{~m} \cdot \mathrm{s}^{-1}$. The velocity resulting from the approximation by the classic Prandtl equation was $v_{\mathrm{C} 1}=8,16 \mathrm{~m} \cdot \mathrm{s}^{-1}$ while the velocity after the approximation by modified Prandtl equation was $v_{\mathrm{C} 2}=7,76 \mathrm{~m} \cdot \mathrm{s}^{-1}$. The difference appears to be significant; therefore we examined how this affects the average velocity. Following the numerically calculated flow rates $Q_{\mathrm{m}}=0,833 \mathrm{~m}^{3} \cdot \mathrm{s}^{-1}, Q_{1}=0,831 \mathrm{~m}^{3} \cdot \mathrm{s}^{-1}$, and $Q_{2}=0,825 \mathrm{~m}^{3} \cdot \mathrm{s}^{-1} \quad$ divided by cross-section area $A_{\text {cir }}=0,126 \mathrm{~m}^{2}$, we obtain average values of velocities which are necessary to determine the Reynolds number: measured $v_{\text {avg m }}=6,63 \mathrm{~m} \cdot \mathrm{s}^{-1}$, approximated by the classic Prandtl equation $v_{\text {avg } 1}=6,61 \mathrm{~m} \cdot \mathrm{s}^{-1}$, and approximated by the modified Prandtl equation $v_{\text {avg } 2}=6,57 \mathrm{~m} \cdot \mathrm{s}^{-1}$. Dispersion of these velocities related to the value of $v_{\mathrm{m}}$ does not exceed $1 \%$.

\subsection{Analytical calculations}

Later in the research, the average velocity for the modified Prandtl distribution was attempted to be determined. The following equation (5) was used for this purpose

$$
v_{\text {avg }}=\frac{4 \int_{0}^{R} v_{\mathrm{C}} \frac{\pi}{2}\left(1-\left(\frac{r}{R}\right)^{2}\right)^{\frac{1}{n}} r \mathrm{~d} r}{A_{\text {cir }}}=\frac{\frac{2 \pi v_{\mathrm{C}}}{R^{\frac{2}{n}}} \int_{0}^{R}\left(R^{2}-r^{2}\right)^{\frac{1}{n}} r \mathrm{~d} r}{\pi R^{2}}
$$

after substituting $\quad z^{2}=R^{2}-r^{2} \rightarrow r \mathrm{~d} r=-z \mathrm{~d} z \quad$ we obtained

$$
v_{\text {avg }}=\frac{-\frac{2 \pi v_{\mathrm{C}}}{R^{\frac{2}{n}}} \int_{R}^{0} z^{2 \frac{1}{n}} z \mathrm{~d} z}{\pi R^{2}}=\frac{-\frac{2 \pi v_{\mathrm{C}}}{R^{\frac{2}{n}}} \int_{R}^{0} z^{2 \frac{1}{n}+1} \mathrm{~d} z}{\pi R^{2}}
$$

and ultimately after the appropriate transformations

$$
v_{\text {avg }}=\frac{n}{n+1} v_{\mathrm{C}}
$$

The above formula shows the fact that the study should determine the dependence of $n=f(R e)$, because the 
exponent $1 / n$ has a decisive influence on the value of the average velocity in the cross-section. It happens very often that it is uncritically accepted as $1 / n=1 / 7$.

Therefore, the average velocity in tested crosssection is $v_{\text {avg2 }}=6,79 \mathrm{~m} \cdot \mathrm{s}^{-1}$ on the basis of a formula (7) for velocity $v_{\mathrm{C} 2}=7,76 \mathrm{~m} \cdot \mathrm{s}^{-1}$ and $n=7$, which is $102.4 \%$ of experimentally determined value. Excess of average velocity may prove that $n$ was too high.

\section{Rectangular cross-section}

Rectangular cross-section with rounded corners is a combination of parts of the rectangular cross-section and circular cross-section. Therefore, this type of ducts was part of compare analysis.

\subsection{The axial velocity distributions}

Experimental study of velocity distribution is made for a rectangular cross-section with width $W=0,5 \mathrm{~m}$ and height $H=0,25 \mathrm{~m}$. The study involved a linear section of the duct composed of 12 same segments of $1 \mathrm{~m}$ length. The profiles were measured on symmetry axes. The traversing step of the thermo-anemometer probe was $\Delta w=\Delta h=4 \mathrm{~mm}$. During the measurements, the same velocity $v_{\mathrm{C}}$ of the air flow at the point of intersection of the two axes should be provided. The test results are shown in the figures Fig. 5 and Fig. 6.

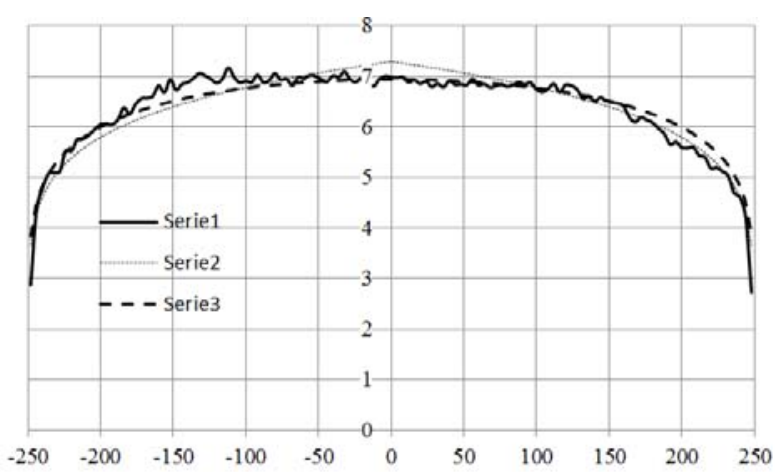

Fig. 5. Velocity distribution along the axis $w$

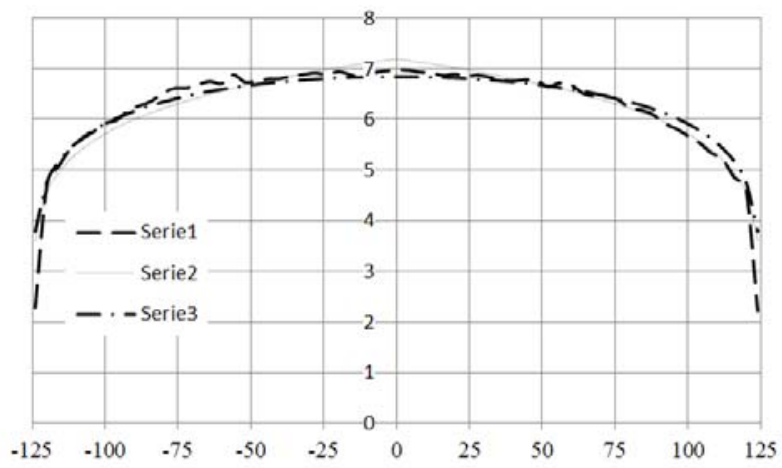

Fig. 6. Velocity distribution along the axis $h$

The figures Fig. 5 and Fig. 6 also present the approximating curves of the measured results by the formulas:

$$
\begin{aligned}
& v_{w}=v_{\mathrm{C}}\left(1-\left(\frac{w}{0,5 W}\right)\right)^{\frac{1}{n}} \text { and } v_{w}=v_{\mathrm{C}}\left(1-\left(\frac{w}{0,5 W}\right)^{2}\right)^{\frac{1}{n}} \\
& v_{h}=v_{\mathrm{C}}\left(1-\left(\frac{h}{0,5 H}\right)\right)^{\frac{1}{n}} \text { and } v_{h}=v_{\mathrm{C}}\left(1-\left(\frac{h}{0,5 H}\right)^{2}\right)^{\frac{1}{n}}
\end{aligned}
$$

Approximating formulas were adopted in the same form as for a circular cross-section. Radius $r$ was replaced by $w$ and $h$ respectively.

Consequently, flow velocity at any point in cross section is given by

$$
v(w, h)=v_{\mathrm{C}}\left(1-\left(\frac{w}{0,5 W}\right)\right)^{\frac{1}{n}}\left(1-\left(\frac{h}{0,5 H}\right)\right)^{\frac{1}{n}}
$$

or

$$
v(w, h)=v_{\mathrm{C}}\left(1-\left(\frac{w}{0,5 W}\right)^{2}\right)^{\frac{1}{n}}\left(1-\left(\frac{h}{0,5 H}\right)^{2}\right)^{\frac{1}{n}}
$$

On the basis of the formula (11), the average velocity $v_{\text {avg }}=5,88 \mathrm{~m} \cdot \mathrm{s}^{-1}$ was calculated by numerical integration and it is necessary to determine the Reynolds number. This result was verified by the method described in Section 3.2.

\subsection{Determining the average velocity using log- Chebyshev method}

The duct is divided into rectangular areas (Fig. 7) by using the log-Chebycheff method. Velocity is measured at corners of these rectangulars.

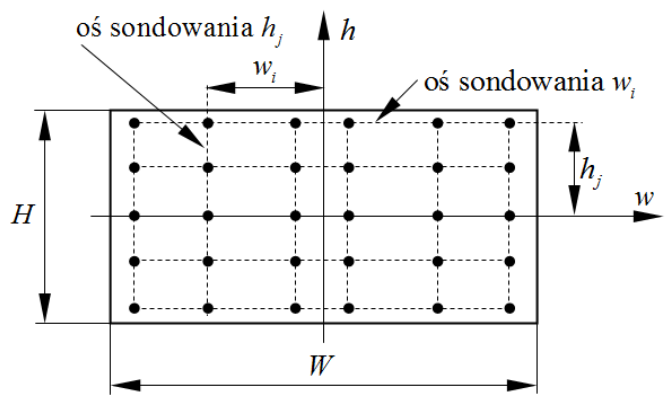

Fig. 7. Example of the measurement points determined in a rectangular cross-section

Table 1. Location of measurement points in a rectangular cross-section [3]

\begin{tabular}{|c|c|}
\hline $\begin{array}{c}\text { Number of traversing } \\
\text { axes } i \text { or } j\end{array}$ & Values $\frac{w_{i}}{W}$ or $\frac{h_{j}}{H}$ \\
\hline 5 & $0, \pm 0,212, \pm 0,426$ \\
\hline 6 & $\pm 0,063, \pm 0,265, \pm 0,439$ \\
\hline 7 & $0, \pm 0,134, \pm 0,297, \pm 0,447$ \\
\hline
\end{tabular}

The points are detrmined by standard ISO 5221 Air distribution and air diffusion - Rules to methods of measuring air flow rate in an air handling duct [3]. The minimum of $25(i=5 ; j=5)$ points must be measured 
in order to get a good average, while the maximum is 49 $(i=7 ; j=7)$ (Table 1). Any intermediate combination is permissible. Velocity measurements were made at various points by using Prandtl tube. For $i=5$ and $j=5$, air velocities obtained at particular points are presented in Table 2 .

Table 2. Coordinates and measurement results for $i=j=5$

\begin{tabular}{|r|r|r|r|r|r|r|}
\hline \multirow{2}{*}{$\begin{array}{c}v \\
\mathrm{~m} \cdot \mathrm{s}^{-1}\end{array}$} & \multicolumn{5}{|c|}{$w_{i} \mathrm{~mm}$} \\
\cline { 2 - 7 } & -213 & -106 & 0 & 106 & 213 \\
\hline \multirow{4}{*}{$\begin{array}{c}h_{j} \\
\mathrm{~mm}\end{array}$} & $-106,5$ & 5,03 & 5,15 & 5,07 & 5,02 & 4,8 \\
\cline { 2 - 7 } & -53 & 5,69 & 6,34 & 6,36 & 6,27 & 5,55 \\
\cline { 2 - 7 } & 0 & 5,75 & 6,87 & 6,95 & 6,68 & 5,96 \\
\cline { 2 - 7 } & 53 & 5,81 & 6,78 & 6,55 & 6,05 & 5,58 \\
\cline { 2 - 7 } & 106,5 & 5,04 & 5,17 & 5,21 & 5,15 & 4,95 \\
\hline
\end{tabular}

On the basis of numerical values contained in Table 2, we obtain $v_{\text {avg }}=5,7512 \mathrm{~m} \cdot \mathrm{s}^{-1}$, which is $97.8 \%$ of value obtained by numerical integration of curves approximated by formula (10).

\subsection{Analytical method}

Velocity distribution defined by the formula (10) can be the basis for analytical determination of the volumetric flow rate

$$
Q=4 v_{\mathrm{C}} \int_{0}^{0,5 W} \int_{0}^{0,5 H}\left(1-\left(\frac{w}{0,5 W}\right)^{2}\right)^{\frac{1}{n}}\left(1-\left(\frac{h}{0,5 H}\right)^{2}\right)^{\frac{1}{n}} \mathrm{~d} h \mathrm{~d} w
$$

The velocity at the duct symmetry axis was adopted $v_{\mathrm{C}}=6,95 \mathrm{~m} \cdot \mathrm{s}^{-1}$, the same as for the numerical integration.

Unfortunately, the integral defined by the formula (11) is not expressed in explicit form. Numerical calculation of this integral can be achieved by using the integral of the special $\Gamma$ function. Using $\Gamma$ function we obtain the following solution of equation (10)

$$
Q=0,25 W H v_{\mathrm{C}}\left(\frac{\Gamma(1 / 2) \Gamma(1+1 / n)}{\Gamma(3 / 2+1 / n)}\right)^{2}
$$

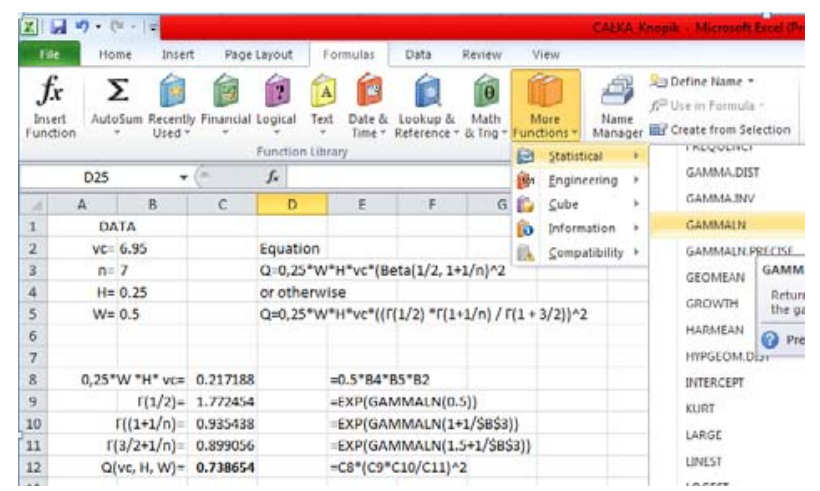

Fig. 8. Solution of the equation (10) using Excel

The numerical value of the flow rate $Q$ from the equation (12) was determined by using Microsoft Excel spreadsheet (Fig. 8).
Due to the GAMMALN command counts $\ln (\Gamma(x))$, it was necessary to enter this function as an internal function of the EXP command. Function $\Gamma(x)$ is determined from the formula

$$
\Gamma(x)=\int_{0}^{\infty} e^{-u} u^{x-1} \mathrm{~d} u
$$

The individual values of the function for the arguments defined by the equation (12) are: $\Gamma(1 / 2) \approx 1,77454$, $\Gamma(1+1 / 7) \approx 0,93438, \Gamma(3 / 2+1 / 7) \approx 0,899056$. where $n=7$. After substituting these values and $W=0,5 \mathrm{~m}$, $H=0,25 \mathrm{~m}, v_{\mathrm{C}}=6,95 \mathrm{~m} \cdot \mathrm{s}^{-1}$ into the equation (12), we received $Q=0,739 \mathrm{~m} \cdot \mathrm{s}^{-1}$, which corresponds to the average velocity $v_{\text {avg }}=5,909 \mathrm{~m} \cdot \mathrm{s}^{-1}$. This is $100.5 \%$ of value obtained during the numerical integration profiles approximated by the equation (10).

\section{Summary}

The analysis shows that the basic form of a power Prandtl formula (1) as well as it modified version (4) can be used for the approximation of the actual velocity distributions.

The classic form of power-law Prandtl is better for circular cross-section, but the modified one is more accurate for rectangular cross-section.

The modified Prandtl formula allows the derivation of a simple relation, which defines the value of the average velocity $v_{\text {avg }}$ as a function of maximum velocity $v_{\mathrm{C}}$ on the symmetry axis of the cross-section.

It is accepted that the modified power-law form Prandtl will be used during testing of rectangular with rounded corners ventilation ducts, because the rectangular cross-section plays a dominant role in this cross-section.

\section{Acknowledgements}

The authors would like to kindly thank the company Nucair Technologies Sp. z o.o., Solec Kujawski, Poland for the performance of tested ducts and the delivery of air supply system.

\section{References}

1. Z., Orzechowski, J. Prywer, R. Zarzycki: Mechanika płynów w inżynierii środowiska (Eng. Fluid Mechanics in Environmental Engineering), WNT, Warszawa, (1997)

2. Y.A Cengel, J.M Cimbala: Fluid Mechanics. Fundamentals and Applications, McGraw-Hill, (2006)

3. Standard ISO 5221: Air distribution and air diffusion - Rules to methods of measuring air flow rate in an handling duct) (1994) 